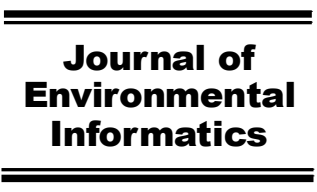

Www.iseis.org/jei

\title{
A Pseudospectral Collocation Approach for Flood Inundation Modelling with Random Input Fields
}

\author{
Y. Huang ${ }^{1}$ and X. S. Qin ${ }^{1,2 *}$ \\ ${ }^{1}$ School of Civil \& Environmental Engineering, Nanyang Technological University, Singapore 639798, Singapore \\ ${ }^{2}$ Environmental Process Modeling Centre (EPMC), Nanyang Environment and Water Research Institute (NEWRI), Nanyang Technological \\ University, Singapore 637141, Singapore
}

Received August 31, 2015; revised December 13, 2015; accepted December 28, 2015; published online August 15, 2016

\begin{abstract}
In this study, an efficient framework of pseudospectral collocation approach combined with the generalized polynomial chaos (gPC) and Karhunen-Loevè expansion ( $\mathrm{gPC} / \mathrm{KLE}$ ) was introduced to examine the flood flow fields within a two-dimensional flood modelling system. In the proposed framework, the heterogeneous random input field (logarithmic Manning's roughness) was approximated by the normalized KLE and the output field of flood flow depth was represented by the gPC expansion, whose coefficients were obtained with a nodal set construction via Smolyak sparse grid quadrature. In total, 3 scenarios (with different levels of input spatial variability) were designed for gPC/KLE application and the results from Monte Carlo simulations were provided as the benchmark for comparison. This study demonstrated that the gPC/KLE approach could predict the statistics of flood flow depth (i.e., means and standard deviations) with significantly less computational requirement than MC; it also outperformed the probabilistic collocation method (PCM) with KLE (PCM/KLE) in terms of fitting accuracy. This study made the first attempt to apply gPC/KLE to flood inundation field and evaluated the effects of key parameters (like the number of eigenpairs and the order of gPC expansion) on model performances.
\end{abstract}

Keywords: collocation, generalized polynomial chaos, Karhunen-Loevè expansion, Smolyak sparse grid, Monte Carlo

\section{Introduction}

Due to scale issues and experimental constraints, it is generally difficult to accurately predetermine model parameters that are spatially distributed, such as data over a two-dimensional computational domain (Wang and Du, 2003; Zhang el al., 2004; Assumaning and Chang, 2014; Yang and Yang, 2014; Liu and Wang, 2015). In flood modelling field, some model parameters may be heterogeneous (i.e., opposed to identical properties in all directions) in space due to complexity of geological formation, such as Manning's roughness. This has led to a wide range of uncertainties (Beven, 2006; Hunter et al., 2007; He et al., 2012; Ahmadi et al., 2015; Li et al., 2015; Chen et al., 2018; Shen et al., 2018). It is desirable that such parameters be treated as random input field(s) and then the related governing equations would be correspondingly turned into stochastic (Aronica et al., 2002; Qin et al., 2008; Huang and Qin, 2014). Over the past years, many stochastic approaches were developped to deal with uncertainties originated from spatial variability in flood inundation modelling (Aronica et al., 2002; Van Vuren et al., 2005; Li et al., 2009; Fan and Huang, 2012;

${ }^{*}$ Corresponding author. Tel.: +65 67905288; Fax: +65 67921650.

E-mail address: xsqin@ntu.edu.sg (X. S. Qin)

ISSN: 1726-2135 print/1684-8799 online

(C) 2017 ISEIS All rights reserved. doi:10.3808.jei.201600339
Yu et al., 2013; Wang et al., 2018; Wu et al., 2018).

The Monte Carlo (MC) approach, as the most commonly used approach based on sampling, can provide solutions to stochastic differential equations (e.g., 2D shallow water equations) in a straightforward and easy-to-implement manner (Ballio and Guadagnini, 2004). However, in order to obtain convergent stochastic results for flood inundation modelling under uncertainty, a relatively large amount of numerical simulations is required, especially for real-world applications; this could bring a fairly high computational cost (Pender and Faulkner, 2011). An alternative is to approximate the random input by Karhunen-Loevè expansion (KLE). In the past few decades, KLE-based stochastic approaches have been widely used in mechanical, groundwater, flood modelling and other non-linear complex systems and processes to deal with heterogeneity of model parameters (Roy and Grilli, 1997; Phoon et al., 2002; Zhang and Lu, 2004; Huang and Qin, 2014a). To represent the corresponding random output field, a stochastic surface response model (SRSM) based on the polynomial chaos expansion (PCE) has been popularly used in various fields (Wiener, 1938; Ghanem and Spanos, 1991; Isukapalli et al., 1998; Li and Zhang, 2007; Li et al., 2011). To obtain polynomial chaos coefficients, the traditional PCE-based scheme uses Galerkin technique to solve a set of coupled equations involving the random normal variables (RNVs) from KLE (Shi et al., 2009). The probabilistic collocation method (PCM) is another type of PCE-based method and 
relatively easier to implement. It is advantageous in the sense that it can obtain PCE coefficients via an inverse matrix scheme and the related methodology would not be influenced by the complexity (non-linearity) of the original numerical modelling systems (Li and Zhang, 2007; Cai et al., 2009; Zhao et al., 2009; Li et al., 2009; Huang et al., 2010; Xiu, 2010).

In recent years, coupled implementation of PCM and KLE (PCM/KLE) has been widely used to deal with problems of uncertainty propagation for numerical models with random input fields (Li and Zhang, 2007; Shi et al., 2009; Huang and Qin, 2014b). The general framework of PCM/KLE involves decomposition of the random input field by KLE and representation of the output field by PCE, by which the complicated stochastic differential equations are transformed into easier-to-solve deterministic equations. The previous applications of PCM/KLE were mainly reported in the fields of ground water modeling and structural modelling systems (Zhang and Lu, 2004; Li and Zhang, 2007; Li et al., 2009; Shi et al., 2010). For example, Li and Zhang (2007) firstly proposed to use KLE to represent the $\log$ transformed hydraulic conductivity field in a porous media, and apply PCM to determine the coefficients of the PCE by solving the hydraulic head fields for different sets of collocation points. The coupled application of PCM/KLE accurately reproduced the statistics of flow field from the corresponding MC simulations using a limited number of numerical simulations. Later on, Shi et al. (2010) used PCM/KLE to study nonlinear flow in heterogeneous unconfined aquifers, with consideration of two types of random inputs that were either correlated or uncorrelated. More recently, Huang and Qin (2014b) made a preliminary test to apply PCM/KLE to quantify uncertainty propagation from a single $2 \mathrm{D}$ random field of floodplain hydraulic conductivity.

To ensure the coefficient matrix of PCE healthy, the PCMrelated methods mostly rely on the regression-based modified collocation approach or the matrix inversion approach (Huang et al., 2007; Xiu, 2010; Fan et al., 2015). The former one aims to have a greater number of collocation points (e.g. 2 or 3 times of the numbers of PCE terms) which would bring additional amount of numerical simulations (Isukapalli et al., 1998; Zheng et al., 2011). The latter one focuses on selecting efficient collocation points to build up full-rank multi-dimensional polynomials, where the efficiency of such a process may be affected by the increasing randomness of KLE approximation for the input field (Shi et al., 2010). In addition, the matrix inversion approach could not guarantee symmetry of the distribution of the collocation points with respect to the origin (Li et al., 2011). Hence, an alternative way of matrix inversion approach is desired to ensure a healthy matrix of PCE coefficients.

As an alternative to PCM, a pseudospectral collocation approach, firstly proposed by Xiu and Krniadakis (2002), has been extensively applied in physical and engineering fields involving stochastic numerical modelling (Xiu and Karniadakis, 2003; Xiu and Hesthaven, 2005; Xiu, 2010). It was an extended version of generalized polynomial chaos (gPC) method based on the stochastic collocation method. Later on, Lin and Tartakovsky (2009) applied this approach coupled with $\mathrm{KLE}$ (gPC/KLE) to solve numerical modelling of three-dimen- sional flows in porous media involving random heterogeneous fields. Another example can be found in Yildirim and Karniadakis (2015), where gPC/KLE was applied in stochastic simulations of ocean waves. The gPC/KLE method is another SR$\mathrm{SM}$ similar to PCM/KLE, of which the coefficients are the approximation of exact gPC coefficients and obtained by nodal construction via Smolyak sparse grid quadrature and a series of repetitive numerical executions for these nodes (Smolyak, 1963; Xiu, 2007; Zhao et al., 2010). Nevertheless, this coupled method has not been applied in the field of uncertainty quantification of flood inundation modelling.

Thus, in this study, we aim to introduce the gPC/KLE method and test its applicability in flood inundation modelling with random input fields. A numerical solver of flood inundation modelling (i.e., FLO-2D Pro) will be embedded into the proposed gPC/KLE framework for a hypothetical case. Then, the accuracy and efficiency of this approach will be examined under the possible effect of two intrinsic parameters of this SRSM, including the number of eigenpairs and the order of gPC expansion. The modelling domain will be involved with different levels of spatial variability, which is characterized by a random Manning's roughness field with a lognormal distribution. We will also compare the performance of gPC/KLE with PCM/KLE in reproducing the statistics of the highly nonlinear fields of flood flows.

\section{Mathematical Formulation}

\subsection{D Flood Problem Formulations}

In this study, we focus on a 2D unsteady-inflow flood inundation modelling problem. The related stochastic governing equations can be described as (O'Brien et al., 1993; FLO2D Software, 2012; Huang and Qin, 2014):

$$
\begin{aligned}
& \frac{\partial h(\mathbf{x})}{\partial t}+\nabla[h(\mathbf{x}) V]=I \\
& \nabla h(\mathbf{x}) g+\frac{n(\mathbf{x})^{2}}{r^{4 / 3}} \frac{\partial V}{\partial t} V|V|=\left(S_{o}-(V \cdot \nabla) V\right) g
\end{aligned}
$$

where $t$ and $r$ are the time [T] and hydraulic radius [L]; $I$ is excess rainfall intensity; $S_{o}$ is the bed slope; $\mathbf{x}(x, y)$ represents the spatial coordinate within a $2 \mathrm{D}$ rectangular modelling domain; $V$ represents the velocity averaged in depth for each specific directions $\mathbf{x}[\mathrm{L} / \mathrm{T}] ; g$ is the gravitational acceleration $\left[\mathrm{L} / \mathrm{T}^{2}\right]$; and all of these above parameters are assumed as deterministic during modelling process. In this study, we define the floodplain Manning's roughness $n(\mathbf{x})$ as a random input with a specific stochastic distribution related to $2 \mathrm{D}$ spatial coordinate $\mathbf{x}$, and place our concern on the output field of flow depth $h(\mathbf{x})$. The $h(\mathbf{x})$ is affected by the input random field $n(\mathbf{x})$ and hence would also be in a stochastic distribution. With these assumptions, equations (1a) and (1b) are transferred into stochastic differential equations (SDEs) and their solutions (i.e., $h(\mathbf{x})$ ) would be described by probability distributions or stochastic moments, including mean and standard deviation (STD). 


\subsection{Approximation of Random Input Field by Normalized Karhunen-Loevè Expansion (KLE)}

In this study, for a 2D modelling domain, we define $\omega \in$ $\Omega$ and $\boldsymbol{x} \in D$, where $\Omega$ and $D$ are the probabilistic space and the measure of the modelling rectangular area, respectively. Let $Z(x, \omega)=\ln n(x)$ be spatially second-order stationary random with mean $\mu_{Z}(\boldsymbol{x})$ and fluctuation $Z^{\prime}(\boldsymbol{x}, \omega)$, showing as: $Z(\boldsymbol{x}, \omega)$ $=\mu_{Z}(\boldsymbol{x})+Z^{\prime}(\boldsymbol{x}, \omega)$, where $Z^{\prime}(\boldsymbol{x}, \omega)$ represents a spatial fluctuation according to its bounded, symmetric, and positive covariance function $C_{Z}\left(\boldsymbol{x}, \boldsymbol{x}^{\prime}\right)=\left\langle Z^{\prime}(\boldsymbol{x}, \omega) Z^{\prime}\left(\boldsymbol{x}^{\prime}, \omega\right)\right\rangle$. The covariance function can be represented by KLE as (Ghanem and Spanos, 1991):

$C_{Z}\left(\boldsymbol{x}_{1}, \boldsymbol{x}_{2}\right)=\sum_{m=1}^{\infty} \lambda_{m} f_{m}\left(\boldsymbol{x}_{1}\right) f_{m}\left(\boldsymbol{x}_{2}\right),(m=1,2, \ldots, \infty$;

$\left.x_{1}, x_{2} \in D\right)$

where $\lambda_{m}$ and $f_{m}(\cdot)$ mean eigenvalues and eigenfunctions, which can be obtained by solving the Fredholm equation (Ghanem and Spanos, 1991). Then, the random input $Z(x, \omega)$ can be represented as:

$Z(\boldsymbol{x}, \omega)=\mu_{Z}(\boldsymbol{x})+\sum_{m=1}^{\infty} \zeta(\omega)\left(\lambda_{m}\right)^{1 / 2} f_{m}(\boldsymbol{x})$

As the random input $Z$ is dependent on the space $D$, use of normalizations (dimensionalizations) are necessary for reducing complexity. For instance, the following normalizations are made in the $\mathrm{x}$ direction: (i) normalized length $\bar{x}=$ $x / L_{x} \in[0,1]$ (where $L_{x}$ is the length of the domain at $x$ direction) and its corresponding normalized correlation length $\eta_{1}=\eta / L_{x}$, (ii) normalized eigenvalues $\lambda=\lambda / L_{x} \in\left[0, \sigma^{2}\right]$ (where $\sigma$ is the STD of $Z$ ), (iii) normalized eigenfunctions $\bar{f}(\bar{x})=f(x) \sqrt{L_{x}}$; hence, we can obtain $\sqrt{\lambda} f(x) \equiv \sqrt{\bar{\lambda}} \bar{f}(\bar{x})$. For y direction, similar normalizations can be made. Subsequently, normalization for a $2 \mathrm{D}$ domain can be obtained based on independent normalizations for both directions. More details about the normalization of KLE can be referred to Zhang and Lu (2004) and Huang and Qin (2014a). A normalized KLE approximation of $Z$ with finite eigenpairs can be expressed as follows (Zhang and Lu, 2004):

$\widehat{Z}=(\overline{\boldsymbol{x}}, \omega)=\mu_{Z}(\overline{\boldsymbol{x}})+\sum_{m=1}^{M} \zeta_{m}(\omega) \bar{g}_{m}(\overline{\boldsymbol{x}})$

where $\bar{g}_{m}(\cdot)$ represent eigenpairs $(\bar{\lambda}, \bar{f}(\cdot)) ; \zeta_{m}(\cdot)$ is the $m^{\text {th }}$ independent standard normal random variable (NRV). According to the theory of KLE, $\sum_{m=1}^{\infty} \bar{\lambda} / \sigma_{Z}^{2}=1$. In order to obtain a more accurate approximation of a certain input random field, more eigenpairs should be kept in the truncated expression of $Z(\cdot)$, and then more energy can be reserved within the random field. However, this would require an additional computation demand (Roy and Grilli, 1997; Shi et al., 2009).

Figure 1 demonstrates how the normalized eigenvalues decay of two different normalized correlation lengths (i.e., 0.5 and 1), and their corresponding cumulative $\bar{\lambda} / \sigma_{Z}^{2}$ are close to 1 when more and more eigenpairs are kept in the approximation and the normalized correlations length is the key factor to determine the decaying rate of eigenvalues and its corresponding cumulative rate. In applying KLE to the stochastic flood modelling system, each KLE item of $Z(\cdot)$ introduces an independent NRV. Therefore, the number of KLE items should be controlled within a suitable range; meanwhile, the energy of KLE approximation of $Z(\cdot)$ would need to be kept sufficient during the modelling process. As our domain of flood modelling system is square-grid, we define $\bar{\eta}_{1}=\bar{\eta}_{2}=\bar{\eta}$ in the rest part of this study, and place more concern on how to represent the Manning's roughness random field with a suitable $\bar{\eta}$. In addition, in a 2D flood modelling system, the spatial complexity in $x$ - and $y$-directions are generally different from each other, which may require different number of the eigenpairs in $x$ - and $y$-directions, respectively (i.e., $M_{x}$ and $M_{y}$ ).
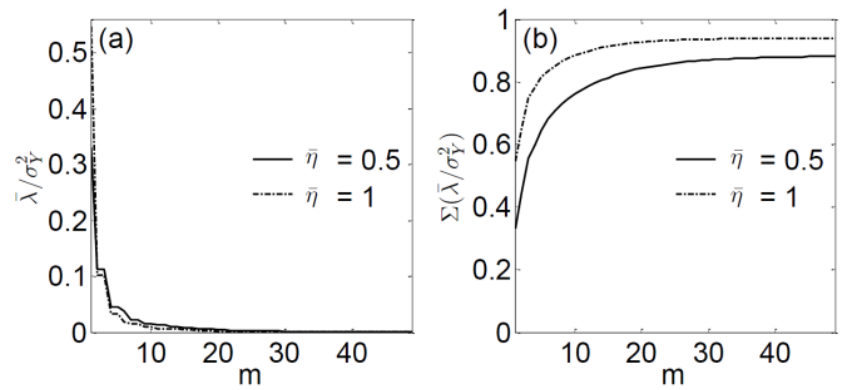

Figure 1. (a) Series of finite $\bar{\lambda}$ for $\bar{\eta}=0.5$ and 1 , and (b) their corresponding cumulative sums for the $2 \mathrm{D}$ modelling domain. Note: the same $\bar{\eta}$ level is selected for both coordinates of the domain

For input parameterization, KLE provides an alternative to represent the heterogeneous fields by involving a finite number of eigenpairs shown in Equation (4). For Gaussian inputs fully characterized by mean and STD, KLE could provide an optimal approximation of the Gaussian processes with/without correlation between random variables. For non-Gaussian inputs, parameterization would be more complicated for KLE and it is beyond the scope of this study (Xiu, 2010). More references on KLE approximation of non-Gaussian inputs can be referred to Phoon et al. (2002), Schwab and Todor (2006), and Li et al. (2007). In this study, we assume a logarithmic Manning's roughness to be Gaussian input and apply KLE to approximate its random field.

\subsection{Construction of gPC Approximation for Output Field}

A combined operation of gPC expansion for the approximation of the output field and Smolyak sparse grid (SSG) quadrature for nodal set construction was firstly proposed by Xiu and Karniadakis (2002) to deal with stochastic numerical modelling system with a high dimensionality of randomness (Xiu, 2007). It has been proved that a low-level gPC expansion for the output fields could reach a high accuracy in terms of gPC simulations (Marzouk et al., 2007; Xiu, 2007; Jakeman et al., 2010). 


\subsubsection{Generalized Polynomial Chaos (gPC)}

After decomposition of random field of logarithmic Manning's roughness as $\hat{Z}(\overline{\mathbf{x}} ; \zeta)$, the stochastic flood modelling system depends on a vector of spatial input variables $\overline{\mathbf{x}} \in[0,1]^{2}$, and an $M$-dimensional vector of NRVs $\zeta=\left\{\zeta_{m}\right\}_{m=1}^{M} \in \mathbb{R}^{M}$, $M \geq 1$. However, we still have little knowledge on the output field of interest (i.e., stochastic moments of flood flows) unless we could solve its corresponding SDEs (i.e., Equation (1)); therefore, we try to use gPC expansion to establish the random functions of the output field. First, let index set $j=\left\{j_{m}\right\}_{m=1}^{M}$ and random space $N^{\text {th }}$-order $M$-dimensional gPC approximation of flood flow field (i.e., maximum flow depth field) be expressed as (Xiu and Karniadakis, 2002):

$\hat{h}_{M}^{N}(\overline{\mathbf{x}} ; \zeta)=\sum_{j=1}^{P} a_{j}(\overline{\mathbf{x}}) \Psi_{j}(\zeta), P=\left(\begin{array}{c}M+N \\ M\end{array}\right)$

where $\Psi_{j}(\cdot)$ represents the $j^{\text {th }}$ orthogonal $M$-dimensional polynomial basis, product of a sequence of corresponding univariate polynomials $\psi_{m}\left(\zeta_{m}\right)$ in each directions of $\zeta_{m}, 1 \leq m \leq M$, which can be expressed as:

$\boldsymbol{\Psi}_{j}(\zeta)=\psi_{j 1}\left(\zeta_{1}\right) \cdots \psi_{j M}\left(\zeta_{M}\right), \sum_{m=1}^{M} j_{m} \leq N$

The expansion coefficients can be obtained as (Xiu and Karniadakis, 2002):

$$
a_{j}=\frac{1}{\gamma_{j}} \mathrm{E}\left[a_{j}(\overline{\mathbf{x}}) \boldsymbol{\psi}_{j}(\zeta)\right]=\frac{1}{\gamma_{j}} \int G(\zeta) \psi_{j}(\zeta) \rho(\zeta) d \zeta
$$

where $\gamma_{j}=\mathrm{E}\left[\boldsymbol{\psi}_{j}^{2}\right]$ are the normalization constants of the orthogonal basis; $\rho$ is the probability density function:

$$
\rho(\zeta)=\prod_{m=1}^{M} \rho_{m}\left(\zeta_{m}\right), \forall p \in \Gamma \subset \mathbb{R}^{M}
$$

where $\Gamma$ is a $M$-dimensional random space. From Equation (5), $a_{j}(\cdot)$ and $\boldsymbol{\Psi}_{j}(\cdot)$ are in pairs but independent to each other. Furthermore, another approximation is made for the exact gPC expansion coefficients $\left\{a_{j}\right\}_{j=1}^{P}$ as (Xiu and Karniadakis, 2002; Xiu, 2007):

$$
\begin{aligned}
\hat{a}_{j} & =\sum_{i=1}^{Q} h\left[Z\left(\zeta^{q}\right)\right] \boldsymbol{\Psi}_{j}\left(\zeta^{q}\right) w^{q} \\
& =\sum_{i=1}^{Q} h\left[n\left(\overline{\mathbf{x}} ; \zeta^{q}\right)\right] \boldsymbol{\Psi}_{j}\left(\zeta^{q}\right) w^{q}, j=1, \cdots, P
\end{aligned}
$$

where $\zeta^{q}$ and $w^{q}$ are the $q^{\text {th }}$ quadrature node and its corresponding weight of an integration rule in the $M$-dimensional random space $\Gamma$, respectively. Herein, sampling in $\Gamma$ is a crucial step in order to get convergent and efficient approximation of gPC expansion coefficients. In this study, $\zeta^{q}$ used in KLE approximation of Manning's roughness are defined as the standard NRVs and the best polynomials basis for them are normal Hermite orthogonal polynomial basis to construct the smooth gPC expansion for the output field $h(\cdot)$.

\subsubsection{Construction of Nodal Sets: Smolyak Sparse Grid (SSG) Quadrature}

As a crucial step in gPC, constructing multi-dimensional nodal sets can be done by several ways, such as full tensor product and Smolyak sparse grid quadrature (Smolyak, 1963; Xiu, 2010). The full tensor product is a more straight-forward way, and its one-dimensional (1D) rule can be shown as (Smolyak, 1963):

$U_{m}^{q_{m}}[h] \equiv \sum_{i=1}^{q_{m}} h\left(\zeta_{m}^{i}\right) w_{m}^{i} \approx \int_{\Omega} h\left(\zeta_{m}^{i}\right) d \zeta_{m}^{i}, m=1, \cdots, M$

The corresponding 1D nodal set is defined as:

$\Xi_{m}^{1}=\left(\zeta_{m}^{1}, \ldots, \zeta_{m}^{q_{m}}\right) \subset \Gamma$

For $M$-dimensional random space $\Gamma$, its full tensor product quadrature can be described as (Smolyak, 1963):

$$
\begin{aligned}
& U^{Q}[h] \equiv\left(U_{1}^{q_{1}} \otimes \cdots \otimes U_{M}^{q_{M}}\right)[h] \\
& =\sum_{i=1}^{q_{1}} \cdots \sum_{i_{M}=1}^{q_{M}} h\left(\zeta_{1}^{i}, \cdots, \zeta_{M}^{i_{M}}\right) \cdot\left(w_{1}^{i_{1}} \otimes \cdots \otimes w_{M}^{i_{M}}\right)
\end{aligned}
$$

where $i_{r}(r=1,2, \ldots, M)$ represents the $r^{\text {th }}$ node index; $q_{r}(r=$ $1,2, \ldots, M)$ represents the number of nodes in the $r^{\text {th }}$ dimension and defined by the node indexes. Obviously, if $q_{r} \equiv q(r=1$, $2, \ldots, M)$, the total amount of full-tensor product nodes $Q$ would be $q^{M}$. As our study involves a high-dimensionality of KLE randomness, the so-called 'curse of dimensionality' would probably be caused by the full tensor product quadrature. Therefore, Smolyak sparse grid (SSG) quadrature, which is specific for (i.e., gPC coefficients) $M$-dimensional random space, can be described as (Smolyak, 1963):

$$
\begin{aligned}
& U^{Q}\left(h_{k, M}\right)=\sum_{k+1 \leq \mathbf{i} \mid \leq M+k}(-1)^{M+k-|\mathbf{i}|} \cdot\left[\begin{array}{l}
M-1 \\
M+k-|\mathbf{i}|
\end{array}\right] \\
& \cdot\left(U_{i_{1}} \otimes \cdots \otimes U_{i_{M}}\right) h\left(\Xi_{M}\right) \\
& |\mathrm{i}|=\sum_{i_{r}=i_{I}}^{i_{M}} i_{r}
\end{aligned}
$$

where $k$ is the level of sparse grid integration; and $M$ represents the random dimensionality of the uncertainty (i.e., the total dimensionality of KLE). The SSG nodal set is defined as (Smolyak, 1963):

$$
\Xi_{M}=\bigcup_{k+1 \leq \mathbf{i} \mid \leq M+k} \Xi_{i_{l}} \otimes \cdots \otimes \Xi_{i_{M}}
$$


In this study, we construct SSG nodal set based on the delayed Genz-Keister basis sequence, which is a full-symmetric interpolatory rule with a Gauss weight function. For more technical details, readers are referred to Genz and Keister (1996). Figure 2 demonstrates a comparison of a two-dimensional $(M=2)$ SSG grid with an accuracy level at $k=3$ and the corresponding full tensor grid, both of which are based on 1D normal Gauss-Hermite grid with $q=9$ quadrature points and polynomial exactness $\tilde{b}=15$ in each dimension.
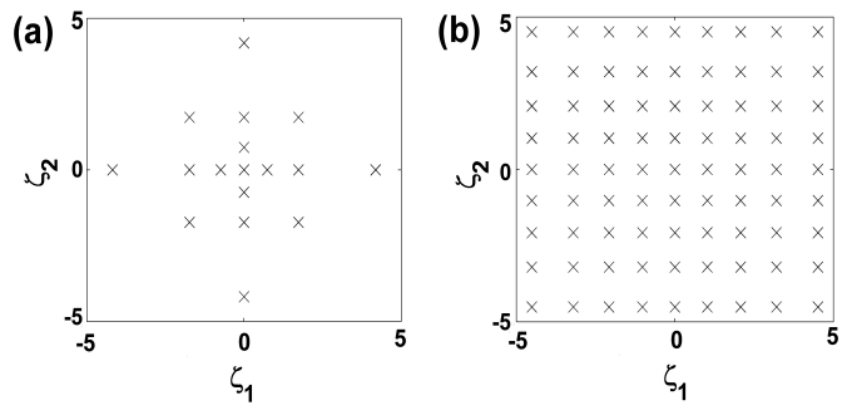

Figure 2. Two nodal constructions based on the GaussHermite grids with delayed Genz-Keister basis sequence: (a) Smolyak sparse grid (SSG), and (b) full tensor grid.

\subsection{Pseudo-spectral Stochastic Collocation Approach Bas- ed on gPC/KLE in Flood Inundation Modelling}

The framework of pseudo-spectral stochastic collocation approach ( $\mathrm{gPC} / \mathrm{KLE})$ for flood modelling system involves the following steps:

(i) Identify the prior distribution (i.e. mean and variance) of $\mathrm{Z}=\ln n$;

(ii) Run a set of Monte Carlo simulations via a flood numerical solver, e.g. FLO-2D Pro (FLO-2D Software 2012) and obtain the mean and STDs of the flood flow field as the benchmark for the proposed gPC/KLE results;

(iii) Choose $\bar{\eta}$ for the specific scenario and the $M$ eigenpairs, $M=M_{x} \times M_{y}$, where $M_{x}$ and $M_{y}$ are the items selected in $\mathrm{x}$ and y directions, respectively. According to Huang and Qin (2014b), the optimal settings of the above parameters are: $\bar{\eta} \in$ $[0.1,5]$ and $M_{x}, M_{y} \in[2,4]$;

(iv) Construct a set of SSG nodal sets $\left\{\zeta^{q}\right\}_{q=1}^{Q}$ by $k$-level (starting from $k=1$ ) SSG quadrature, and then transform them into the corresponding random field of Manning's roughness as $\left\{Z_{i}\left(\overline{\mathbf{x}} ; \zeta^{q}\right)\right\}_{q=1}^{Q}$ over the 2D modelling domain; substitute them into Equation (1a-b), which could be solved by the flood numerical solver; finally, build up a matrix of the correspondding gPC expansion coefficients $\left\{\hat{a}_{j}\right\}_{j=1}^{M}$, which is the stochastic surface response model;

(v) Select a set of $P$ collocation points for a given $\operatorname{order} N$; build up their corresponding $\left\{\boldsymbol{\Psi}_{j}(\zeta)\right\}_{j=1}^{P}$ and calculate the flood -flow mean and STD based on the following equations ( $\mathrm{Li}$ and Zhang, 2007; Shi et al., 2009):

Mean: $\langle\hat{h}\rangle=\hat{a}_{1}(\boldsymbol{x})$
STD: $\sigma_{h}(\boldsymbol{x})=\sqrt{\sum_{j=2}^{P} \bar{a}_{j}^{2}(\overline{\boldsymbol{x}})\left\langle\boldsymbol{\psi}_{j}^{2}\right\rangle}$

(vi) Evaluate the efficiency and accuracy of gPC/KLE. To quantitatively assess the accuracy of gPC approximation of flood flow field, the root means squared error (RMSE) and coefficient of determination $\left(R^{2}\right)$ are used (O'Connell et al., 1970; Karunanithi et al., 1994; Yu et al., 2014).

(vii) Repeat the operation from step (ii) to step (v) until an optimal SRSM is found.

The distinct advantage of this framework is that, unlike PCM/KLE, it establishes the PC expansion matrix not by solving its corresponding Vandermonde matrix, but by using an approximation based on another projection via SSG quadrature.

There are a number of sensitive parametric factors that would affect the efficiency of implementing the mentioned method. For KLE approximation of input fields, the normalized correlation length and the number of KLE items are sensitive parameters. For PCE approximation of the output field, the Smolyak's sparse grid (SSG) level, the order of PCE expansion, and the construction rule for SSG nodes (e.g. full tensor construction, Gauss-Hermite rule, or Genz-Keister rule) would notably influence the results. In addition, some extrinsic factors may also affect the efficiency of gPC/KLE application, such as the spatially variability, grid size, inflow hydrograph and other boundary conditions. In this study, we focus on two intrinsic parameters (including the number of KLE items and the order of gPC expansion) and one extrinsic factor (i.e. spatial variability) as they are considered most sensitive based on our test. Other potentially sensitive parameters could be considered in further studies.

\section{Illustrative Example}

\subsection{Configuration of Study Case}

A simple 2D flood inundation case (with an area of $228 \mathrm{~m}^{2}$ ) under the dual effect of unsteady-inflow and rainfall is introduced (FLO-2D Software, 2012). The peak discharge for the unsteady-inflow hydrograph is $393.55 \mathrm{~m}^{3} / \mathrm{s}$ and the total rainfall is $236.2 \mathrm{~mm}$. The study area is a typical conically-shaped desert alluvial with multiple distributary channels and is discretized into $31 \times 33$ rectangular domain. In this study, $\mathrm{X}$ (in $\mathrm{X}$ direction) and $\mathrm{Y}$ (in $\mathrm{y}$-direction) are denoted as the normalized coordinates (with $0 \leq \mathrm{X}, \mathrm{Y} \leq 1$ ). More details about this study case can be referred to FLO-2D Software (2012) and Huang and Qin (2014b). Three modeling scenarios (i.e. Scenarios 1, 2 and 3 ) are designed to validate the introduced gPC/KLE approach, which are of different variances of the $2^{\text {nd }}$-order stationary Gaussian random fields for logarithmic Manning's roughness. Scenarios 1, 2, and 3 have the same mean (i.e. $\langle Z\rangle=$ $-3.0)$, but their variances $\left(\sigma_{Z}^{2}\right)$ are set to $0.09,0.01$ and 0.25 , respectively. Due to the existence of multiple distributary channels within the 2D modelling domain, the geological formation leads to stochastic asymmetry of random-input distributions 
(i.e., Manning's roughness); as a consequence, the complexity of the random inputs in $\mathrm{x}$-direction is higher than that in $\mathrm{y}$ direction. Therefore, 12 SRSMs with different levels of complexities (i.e. with different eigenpairs used in $\mathrm{x}$ and $\mathrm{y}$ directions) are designed to tackle the random fields. The statistics, especially the standard deviation (STD) of the flow field simulated by the above established SRSMs, are compared with those obtained from MC simulations. To ensure convergence, 5000, 5000, and 10,000 runs for Scenarios 1, 2 and 3, respectively, are adopted. The MC simulation results are considered as 'true' moments and taken as the benchmarks. Table 1 shows the SRSMs used in this study. They include: (i) 6 gPC/KLE SRSMs (M1 to M6) for Scenario 1, (ii) 3 (M7 to M9) for Scenario 2, (iii) 3 SRSMs (i.e., M10 to M12) for Scenario 3, and (iv) 2 SRSMs (i.e., M13 and M14) for Scenario 1, which are based on PCM/KLEs and used to compare with SRSMs M1 and M2. The first 12 SRSM models (M1-M12) are shortlisted based on performances of a large number of SRSMs (more than 200) tested under each scenario.

\subsection{Effect of Parameters Related to the gPC/KLE Appro- ximations}

In Scenario 1, the coefficient of variance for the random field is $\sigma_{n} /\langle n\rangle=0.307$. The SSG level and the normalized correlation length are set as $3^{\text {rd }}$ and 0.5 , respectively. These settings are based on tests using different SSG levels and correlation lengths. The definitions of these parameters are referred to Chow et al. (1988). After a few tests, the $3^{\text {rd }}$-order gPC/KLE model with the $3^{\text {rd }}$-level SSG (M2) is considered as the best SRSM for Scenario 1. Figure 3 shows two realizations of the random field of Manning's roughness corresponding to: (a) the $15^{\text {th }}$ SSG node, and (b) the $35^{\text {th }}$ collocation point for the $3^{\text {rd }}$ order gPC/KLE approximation of the flow depth field with the $3^{\text {rd }}$-level SSG (M2), respectively. The two realizations are from two different collocation point systems.

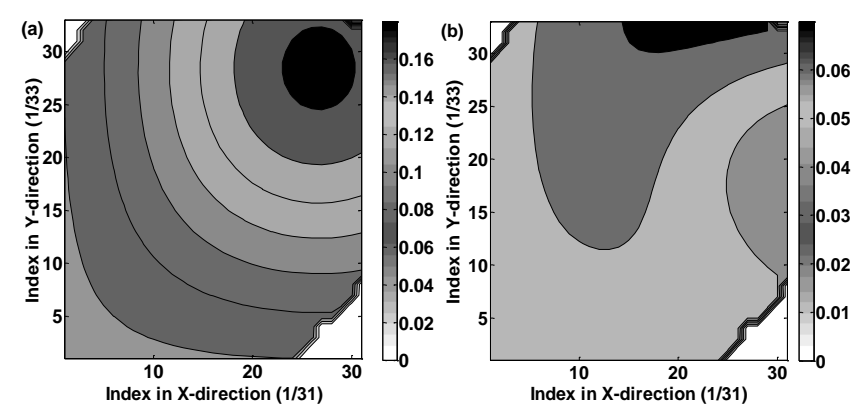

Figure 3. Example realizations of random field of Manning's roughness over the modelling domain under Scenario 1 at (a) the $15^{\text {th }} \mathrm{SSG}$ node, and (b) the $35^{\text {th }}$ collocation point of the $3^{\text {rd }}$-order gPC/KLE with the $3^{\text {rd }}$-level SSG, respectively. Note: The modelling domain is divided into 31 (in X-direction) $\times 33$ (in Y-direction) grid elements; the $2 \mathrm{D}$ covariance function for the random field $n(\mathrm{x})$ corresponds to the normalized correlation length $\bar{\eta}=0.5$ and $M=6 \mathrm{KLE}$ items.

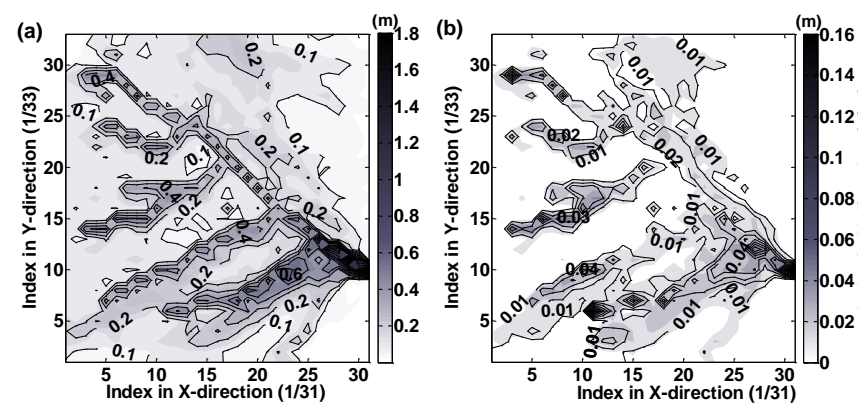

Figure 4. Comparison of statistics of the flow depth between the gPC/KLE and MC methods: (a) mean, and (b) STD.

Note: The grey contour map is the result from MC simulation and the solid lines are from the 3rd-order gPC/KLE model with the $3^{\text {rd }}$-level SSG construction (M2) for Scenario 1.

Table 1. Fuzzy Parameters of Economic Data $\left(\$ / \mathrm{m}^{3}\right)$ and Seasonal Flows $\left(10^{3} \mathrm{~m}^{3}\right)$.

\begin{tabular}{|c|c|c|c|c|c|c|c|c|}
\hline Type & Scenario & SRSM & $\eta$ & $\mathrm{M}\left(\mathrm{M}_{\mathrm{x}} \times \mathrm{M}_{\mathrm{y}}\right)$ & $\mathrm{k}$ & $\mathrm{N}$ & Q & $\mathrm{P}$ \\
\hline \multirow[t]{12}{*}{ gPC/KLE } & \multirow[t]{6}{*}{1} & M1 & 0.5 & $4(2 \times 2)$ & 3 & 3 & 81 & - \\
\hline & & M2 & 0.5 & $6(2 \times 3)$ & 3 & 3 & 257 & - \\
\hline & & M3 & 0.5 & $8(2 \times 4)$ & 3 & 3 & 609 & - \\
\hline & & M4 & 0.5 & $9(3 \times 3)$ & 3 & 3 & 871 & - \\
\hline & & M5 & 0.5 & $6(2 \times 3)$ & 3 & 2 & 257 & - \\
\hline & & M6 & 0.5 & $6(2 \times 3)$ & 3 & 4 & 257 & - \\
\hline & \multirow[t]{3}{*}{2} & M7 & 0.5 & $4(2 \times 2)$ & 2 & 2 & 33 & - \\
\hline & & M8 & 0.5 & $4(2 \times 2)$ & 2 & 3 & 33 & - \\
\hline & & M9 & 0.5 & $4(2 \times 2)$ & 2 & 4 & 33 & - \\
\hline & \multirow[t]{3}{*}{3} & M10 & 0.5 & $8(2 \times 4)$ & 3 & 2 & 609 & - \\
\hline & & M11 & 0.5 & $8(2 \times 4)$ & 3 & 3 & 609 & - \\
\hline & & M12 & 0.5 & $8(2 \times 4)$ & 3 & 4 & 609 & - \\
\hline \multirow[t]{2}{*}{ PCM/KLE } & \multirow[t]{2}{*}{1} & M13 & 0.5 & $6(2 \times 3)$ & - & 2 & - & 28 \\
\hline & & M14 & 0.5 & $6(2 \times 3)$ & - & 3 & - & 84 \\
\hline
\end{tabular}


Figure 4 shows the simulated mean and STD of flood flow fields from M2 and MC simulations. M2 requires only $257 \mathrm{nu}-$ merical executions based on the nodal set from SSG construction; it can closely capture the statistics of the simulated depth field from MC with 5,000 runs. For instance, the mean and STD of flow depth at grid $(31 / 31,10 / 33)$ (the peak values within the entire domain) simulated by M2 are 1.8255 and $0.1616 \mathrm{~m}$, respectively; those from $\mathrm{MC}$ are about 0.0109 and $3.32 \%$ lower (i.e. 1.8253 and $0.1564 \mathrm{~m}$, respectively). For all gPC/KLE (M1 to $\mathrm{M} 12$ ) results, the means are found consistently close to those from MC. Hence, in the followed discussions, the performance of STD will be focused on. In the next two sections, we try to examine the effect of the three parameters on the prediction performance. They include the number of eigenpairs, the order of $\mathrm{gPC} / \mathrm{KLE}$ approximation, and the spatial variability.

\subsubsection{Effect of the Number of Eigenpairs Kept for Normalized} KLE

To test the effect of the number of eigenpairs kept in the xand $y$ - directions of the rectangular modelling domain (i.e., $M_{x}$ and $M_{y}$ ), 4 gPC/KLE models (i.e. M1 to M4) are designed with 4, 6, 8 and 9 eigenpairs $\left(M=M_{x} \times M_{y}\right)$, which could help keep $59.3,65.4,68.4$, and $72.0 \%$ energy within the random Manning's roughness field, respectively. To obtain the correspondding gPC/KLE approximation for the output field of flood-flow depths, four SSG nodal sets (with 81, 237, 609 and 871 nodes) are constructed and then the corresponding random Manning's roughness fields are obtained by involving a series of numerical executions via FLO-2D Pro solver. The results at six locations, with $\mathrm{X}$ being 10/31, 17/31 and 30/31 and $\mathrm{Y}$ being 10/33, 17/33 and 30/33, are chosen for analysis; these locations are from the upstream, middlestream and downstream in both $\mathrm{x}$ and $\mathrm{y}$ directions.

Figures 5(a) and 5(b) present the RMSE and the $\mathrm{R}^{2}$ of STDs fitting for the six locations, respectively. Both the errors and the determination coefficients are plotted against the number of eigenpairs (corresponding to the models M1-M4). Firstly, it can be found that the RMSEs in all the locations would decrease slightly and then increase with the increase of the number of eigenpairs (>6), and the trend of $R^{2}$ is opposite. When the number of eigenpairs is 6 (i.e. M2), the RMSE and $\mathrm{R}^{2}$ achieve their best values for all profiles. This may because the selection of ratio of $M_{y}$ to $M_{x}$ for M2 (i.e. $M_{y} / M_{x}=3 / 2=1.5$ ) is more appropriate for this specific modeling domain. Secondly, the performance of different models shows large variations along different profiles. For the $x$ coordinate, the highest accuracy of STD fitting under the same model is found for the profile $\mathrm{X}=30 / 31$ where the average RMSE and $\mathrm{R}^{2}$ (for M1-M4) are $0.003 \mathrm{~m}$ and 0.998 , respectively; the poorest performer is found at profile of $X=17 / 31$, where RMSE ranks in the middle (i.e. $0.009 \mathrm{~m}$ ) and the $\mathrm{R}^{2}$ ranks the lowest (i.e. 0.782); the profile $X=10 / 31$ shows better performance than $X=17 / 31$, but the error becomes more significant when the number of eigenpairs is above 6 . For the $y$ coordinate, the profile $\mathrm{Y}=$ $30 / 33$ also shows a better result (i.e. average RMSE is 0.004 and average $\mathrm{R}^{2}=0.930$ over different numbers of eigenpairs) than others; while those at $Y=10 / 33$ and $Y=17 / 33$ illustrate similar inferior performances. The notable spatial variations in terms of STD fitting may because of the existence of multiple distributary channels in the 2D modelling domain (as shown in Figure 4). For instance, the profiles of $X=30 / 31$ and $Y=30 / 33$ are characterized by almost single channel conditions, and profiles along the upper and middle parts of the domain show much higher complexity of topographical and morphological conformations. From Figure 4, the flow depth contours along the $y$ direction are more heterogeneously distributed than those along the $x$ direction.
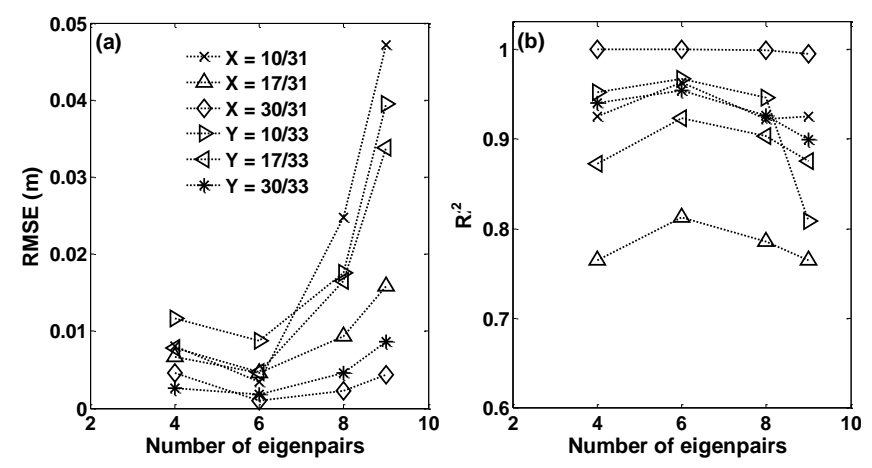

Figure 5. Evaluation of STD fitting in terms of (a) RMSE and (b) $\mathrm{R}^{2}$ for gPC/KLE with different numbers of eigenpairs at six locations of concern. Note M1, M2, M3 and M4 are built up with 4, 6, 8 and 9 eigenpairs, respectively.
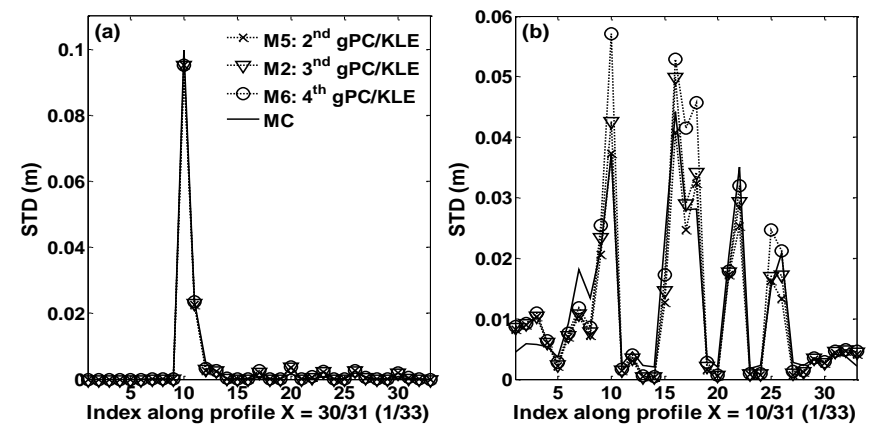

Figure 6. Comparison of STDs of flow depths simulated by the $2^{\text {nd }}-, 3^{\text {rd }}-$, and $4^{\text {th }}$-order gPC/KLE models and MC simulation along the cross-section profiles of (a) $\mathrm{X}=30 / 31$ and (b) $X=10 / 31$, respectively.

\subsubsection{Effect of the Order of gPC Expansion}

To explore the effect of the order $(N)$ of gPC expansion on the efficiency of approximating the highly nonlinear flows over the rectangular domain, three gPC/KLE models including M5 ( $2^{\text {nd }}$-order), M2 ( $3^{\text {rd }}$-order), and M6 ( $4^{\text {th }}$-order) are established. M5 and M6 are set up with the same level of SSG construction as M2. Figure 6 compares the STDs of the flow depths in Scenario 1 , simulated by the $2^{\text {nd }}, 3^{\text {rd }}, 4^{\text {th }}$-order $g P C / K L E$ models and the MC method along the cross-section profiles of $\mathrm{X}=$ $30 / 31$ (single channel) and $X=10 / 31$ (multiple channels), respectively. It is indicated that, for the single-channel condition, M5, M2, and M6 reproduce almost identical STD results as the MC method (i.e. the corresponding RMSE and the $\mathrm{R}^{2}$ are 0.001 $\mathrm{m}$ and 0.999, respectively). For multiple-channel condition, Figure 6(b) shows that when STD of the flow depth is below 
$0.02 \mathrm{~m}$, all of the three orders of gPC/KLE models would lead to similar results as obtained from MC simulation; whereas, for the high peak flow depths, the $2^{\text {nd }}$ - and the $3^{\text {rd }}$ - order models (i.e. M5 and M2) outperform the $4^{\text {th }}$-order one (i.e. M6). For instance, the simulated STDs in grid element $(10 / 31,10 / 33)$ are $0.0235,0.0251,0.0343 \mathrm{~m}$ by the $2^{\text {nd }}-, 3^{\text {rd }}-$, and $4^{\text {th }}$-order models, respectively.

\subsubsection{Effect of Spatial Variability}

In this section, the effect stemming from the spatial variability is analysed. Scenarios 1 to 3 are designed with $\sigma_{Z}^{2}$ levels at $0.09,0.01$, and 0.25 , respectively. The result from Scenario 1 has been demonstrated in the previous sections. For Scenario 2 , based on our tests on various combinations, the following optimal parameter settings are employed: (i) the coefficients matrix of gPC/KLE is built up based on the $2^{\text {nd }}$-level SSG; (ii) the correlation length is set as 0.5 ; and (iii) the number of eigenpairs are selected as $M=M_{x} \times M_{y}=2 \times 2=4$. MC simulations are based on 5,000 runs of numerical solver for benchmarking. Under Scenario 2, $3 \mathrm{gPC} / \mathrm{KLE}$ models with 3 different orders (M7, M8, and M9 as listed in Table 1) are established to generate the flood flow field.

Figure 7 shows the comparison of STDs of flow depths simulated by the $2^{\text {nd }}-, 3^{\text {rd }}-$, and $4^{\text {th }}$-order gPC/KLE models (M7, M8, and M9) and MC simulations for Scenario 2. In total, 4 different profiles within the modeling domain are selected, including $X=10 / 31, X=30 / 31, Y=10 / 33$, and $Y=30 / 33$. It appears that the performances of STD simulations are satisfactory, except for somewhat fluctuations of accuracy from models with different orders. For example, the RMSE of STD fitting for M7, M8, and M9 along the profile $X=10 / 31$ are $0.0036,0.027$ and $0.002 \mathrm{~m}$, respectively; and the corresponding $\mathrm{R}^{2}$ are $0.9547,0.9624$ and 0.9442 . Comparing with the performances of models in Scenario 1, those in Scenario 2 are found comparable. For example, the average RMSE value over the $2^{\text {nd }}-, 3^{\text {rd }}$-, and $4^{\text {th }}$-order gPC/KLE models (i.e. M5, M2, and M6) along the profile $X=10 / 31$ in Scenario 1 is about $0.00175 \mathrm{~m}$ lower than that in Scenario 2; the average $\mathrm{R}^{2}$ of the three models in Scenario 1 (i.e. 0.954) is slightly higher than that in Scenario 2 (i.e. 0.949). This demonstrates that 2 eigenpairs for each coordinate is sufficient to reflect the spatial variability for Scenario 2. Hence, the gPC/KLE model can be constructed using a lower number of SSG nodes (i.e. lower computational requirement) for less complex spatial conditions.

Figure 8 shows the simulated STDs of the flow depths by gPC/KLE models with three orders $\left(2^{\text {nd }}\right.$ for M10, $3^{\text {rd }}$ for M11, and $4^{\text {th }}$ for M12) for Scenario 3. The number of SSG nodes is set as 609 and the MC simulations are based on 10,000 runs. Similar to M7 - M9, the STDs from M10 - M12 along the profile of $X=30 / 31$ (i.e. single channel) are almost identical. However, for the profiles with a higher complexity (e.g. more than 3 channels as shown in Figures 8a and 8d), higher errors of fitting are found compared with those from Figures $7 \mathrm{a}$ and $7 \mathrm{~d}$. For example, along the profile $X=10 / 31$, the deviation of the simulated STD would increase significantly (i.e. RMSE increases from 0.0108 to $0.326 \mathrm{~m}$, and $\mathrm{R}^{2}$ decreases from 0.892 to 0.872 ) with the increase of order (from $2^{\text {nd }}$ to $4^{\text {th }}$ ). Particularly, the errors of STD fitting on peaks have larger deviations. For example, at grid element of $(10 / 31,10 / 33)$ for Scenario 3 (as shown in Figure 8a), the STDs are 0.0051, 0.0189, and 0.0696
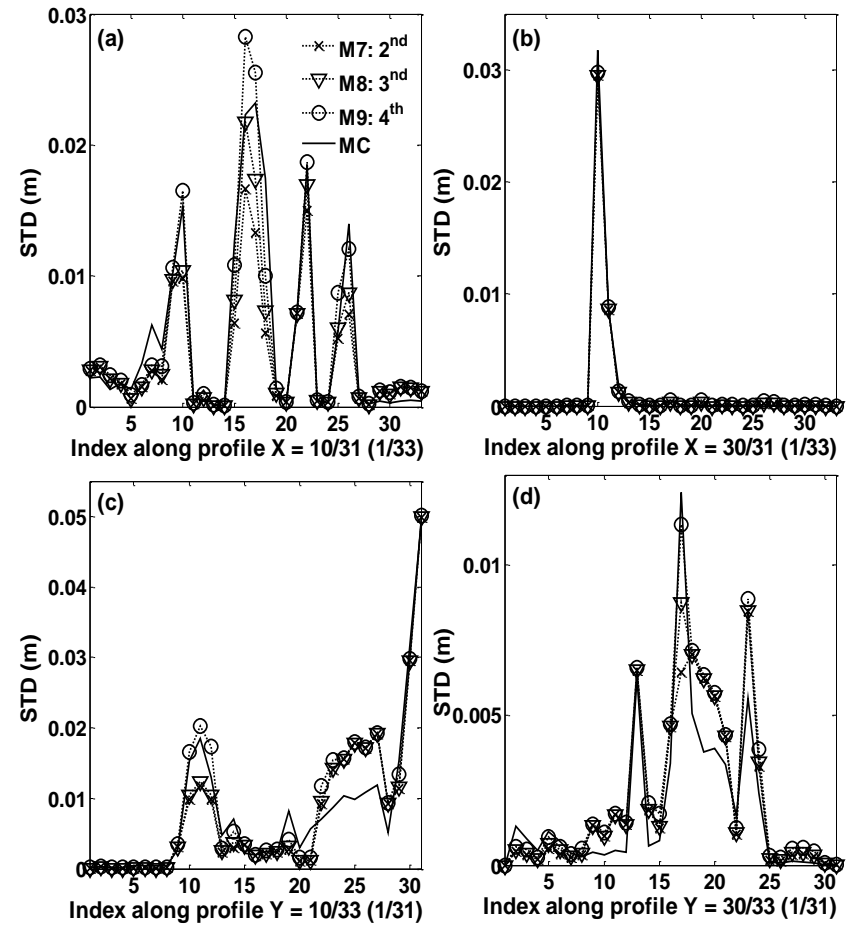

Figure 7. Comparison of STDs of flow depths simulated by the $2^{\text {nd }}-, 3^{\text {rd }}-$, and $4^{\text {th }}$-order gPC/KLE and MC simulations for Scenario 2 along the cross-section profiles of: (a) $X=10 / 31$;

(b) $\mathrm{X}=30 / 31$; (c) $\mathrm{Y}=10 / 33$; (d) $\mathrm{Y}=30 / 33$.
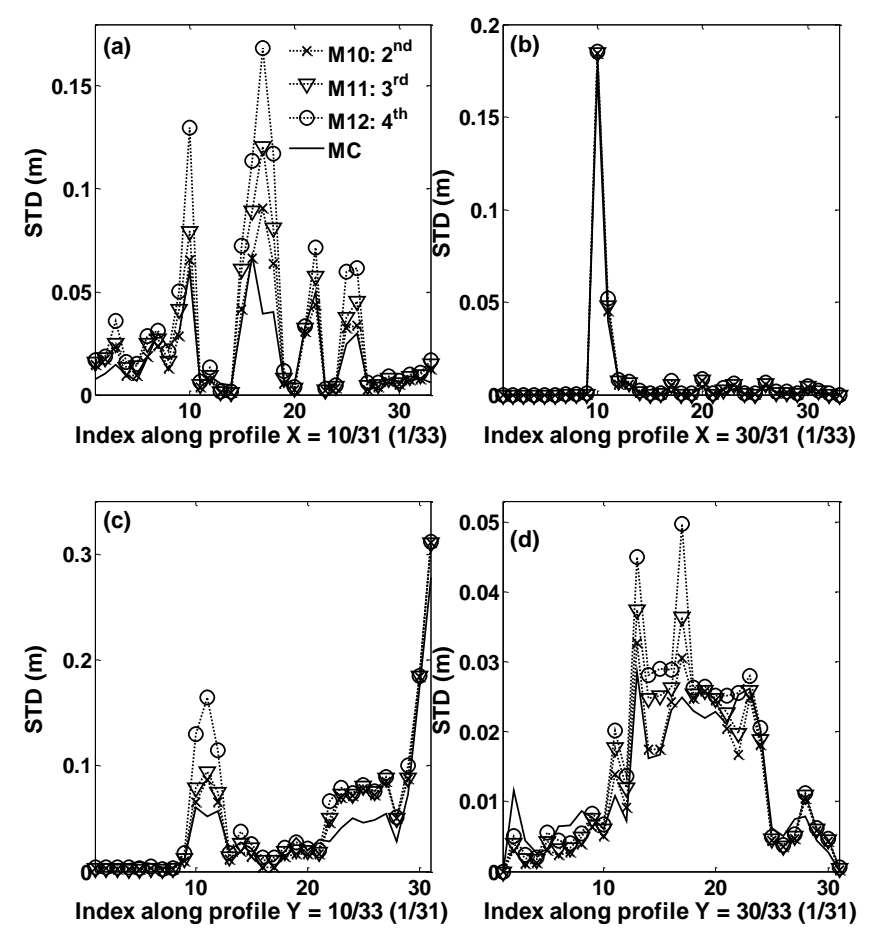

Figure 8. Comparison of STDs of flow depths simulated by 
gPC/KLE and MC methods for Scenario 3 along the crosssection profiles of: (a) $\mathrm{X}=10 / 31$; (b) $\mathrm{X}=30 / 31$; (c) $\mathrm{Y}=$ $10 / 33$; (d) $Y=30 / 33$.

$\mathrm{m}$ higher than that from MC simulation (i.e. $0.0603 \mathrm{~m}$ ) for M10, M11, and M12; while for Scenario 1 (as shown in Figure 6b), the differences are $0.00005,0.0053$ and $0.0198 \mathrm{~m}$ for M5, M2, and M6, respectively. Hence, the spatial variability associated with input random field is linked with the fitting performance of the gPC/KLE model. Generally, the greater the variability, the higher the fitting error. It is also noted that, at the same $\sigma_{Z}^{2}$ level, the order of gPC approximation could also cause considerable effect on fitting performance. This implies that the order can be taken as a more operable tool in fine-tuning the gPC/KLE model to achieve a higher accuracy, compared with the number of eigenpairs and the SSG levels; this is because the change of order would not bring additional runs of the numerical solver (i.e. FLO-2D).

\subsection{Further Discussions}

To further demonstrate the advantage of the introduced method, we compared gPC/KLE with another popular probabilistic collocation method, namely PCM/KLE. PCM/KLE has been applied to deal with the field of $2 \mathrm{D}$ flood modelling system with nonlinear flood flow under uncertainty (Huang and Qin 2014b). Herein, the $2^{\text {nd }}$ - and $3^{\text {rd }}$-order gPC/KLE models (i.e. M1 and M2) under Scenario 1 are used for comparison. Correspondingly, the $2^{\text {nd }}$ - and $3^{\text {rd }}$-order PCM/KLE models (i.e. M13 and M14 as shown in Table 1, respectively) are established under the same scenario, with 6 eigenpairs being adopted and the normalized correlation length being set as 0.5. Figure 9 presents the simulated STDs from two models (i.e. M1 vs. M13 and M2 vs. M14) and MC simulations at different locations within the modelling domain.

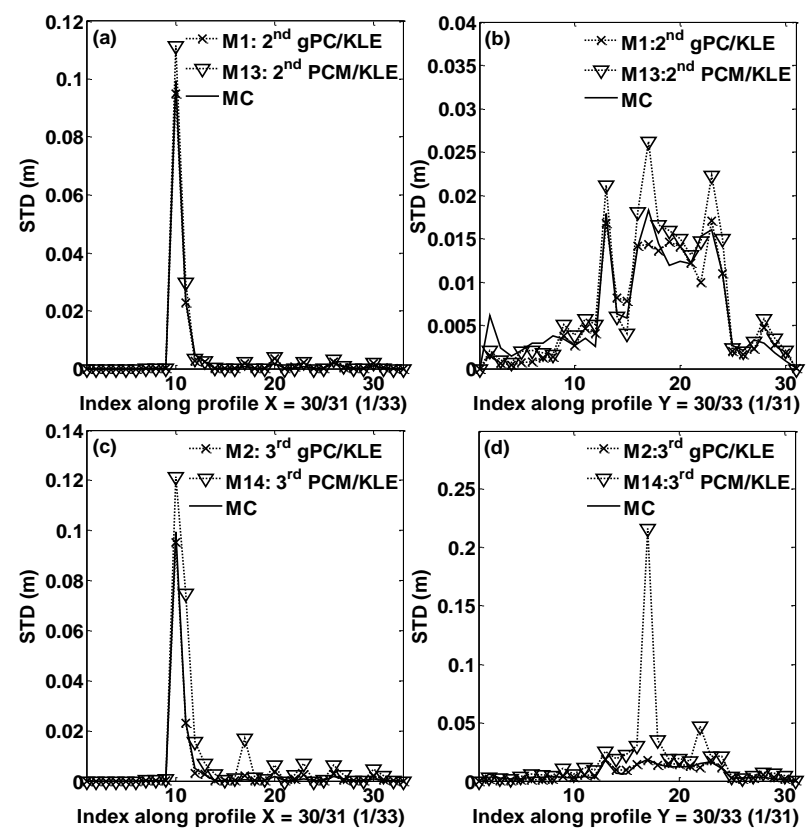

Figure 9. Comparisons of STDs of flow depths between gPC/KLE and PCM/KLE models: (a) the $2^{\text {nd }}$ order modelling along profile $X=30 / 31$; (b) the $2^{\text {nd }}$ order modelling along profile $\mathrm{Y}=30 / 33$; (c) the $3^{\text {rd }}$ order modelling along profile $\mathrm{X}$ $=30 / 31 ;$ (d) the $3^{\text {rd }}$ order modelling along profile $\mathrm{Y}=30 / 33$.

Figures $9 \mathrm{a}$ and $9 \mathrm{~b}$ illustrate the $2^{\text {nd }}$-order comparison. For simple channel condition (like single channel), the STD from PCM/KLE (M13) is slightly higher than those from gPC/KLE and MC. For more complicated profile (like multiple channels at $\mathrm{Y}=30 / 33$ ), the PCM/KLE model has a few obvious overestimations at some peaks. Overall, the RMSEs for M13 and M1 are 0.0027 and $0.0019 \mathrm{~m}$, respectively. From Figures 9c and 9d, the STD reproductions from PCM/KLE show a much higher overestimation for both single and multiple channel conditions. For example, the STD values at the grid element (17/31, 30/33) simulated by MC, gPC/KLE and PCM/KLE are $0.0183,0.0176$ and $0.216 \mathrm{~m}$, respectively. The reason may be that, building up the $3^{\text {rd }}$-order full-rank matrix of the Hermite polynomials requires an efficient selection of collocation points from the roots of the $4^{\text {th }}$-order Hermite polynomial $[-\sqrt{3+\sqrt{6}}$, $\sqrt{3+\sqrt{6}},-\sqrt{3-\sqrt{6}}, \sqrt{3-\sqrt{6}}]$. However, such a root set does not include ' 0 ' that captures the highest probability region for Gaussian random field, which could lead to an inferior performance of the $3^{\text {rd }}$-order PCM/KLE compared with the $2^{\text {nd }}$-order one (Li and Zhang, 2007; Li et al., 2011).

Comparing with PCM/KLE, a significant advantage of $\mathrm{gPC} / \mathrm{KLE}$ is that, to obtain an accurate $\mathrm{gPC} / \mathrm{KLE}$ approximation of flood flow field (in Equation 5), we can express the random input(s) using the analytical polynomial formula as shown in Equation 6. Subsequently, the gPC expansion coefficients $\left\{a_{j}\right\}_{j=1}^{P}$ (in Equation 7) are obtained based on a finite number of fixed values of SSG nodes (i.e. roots of higher order polynomial). This treatment can effectively avoid the difficulty in applying the inverse matrix approach (as adopted in a normal PCM/KLE framework) to complex problems with a high dimension of randomness and a large number of KLE items. Such a difficulty is brought about from construction of a full-rank Vandermonde-like coefficient matrix (i.e. a set of given-order orthogonal polynomials) and computation of its inverse (Xiu, 2007; Li et al., 2011). Hence, a relatively high veracity in reproducing the statistics of the non-linear flood flow field can be achieved at a much lower computational cost compared with traditional MC simulation. For instance, M3 is built up with 257 numerical executions of FLO-2D solver. The STD simulated from M3 well reproduced the result from MCS with 5,000 numerical executions. The computational cost has saved by about $95 \%$.

The spatial variability in the $\mathrm{x}$ and $\mathrm{y}$ directions would bring different effects on the predicted STDs of the flood flows. This is especially true for those multi-channel conditions (i.e. asymmetric geological conditions), which is common in real flood modeling process. To tackle such a complexity, it is necessary to use different numbers of eigenpairs for different directions (i.e. $M_{x}$ and $M_{y}$ kept for X- and y-direction, respectively) within the modeling domain. When $\sigma_{Z}^{2}$ is small enough, such as 0.01 adopted in Scenario 2, the effect of the geological asy- mmetry 
becomes negligible, and there is no need to consider the difference between $M_{x}$ and $M_{y}$.

\section{Conclusions}

In this study, a pseudospectral collocation approach coupled with the generalized polynomial chaos and KarhunenLoevè expansion (gPC/KLE) for flood inundation modelling with random input fields was introduced. The gPC/KLE framework enabled accurate and efficient approximation of the nonlinear flood flows with specific input random fields, while avoiding construction of the Vandermonde-like coefficient matrix adopted in a normal PCM/KLE approach. Three scenarios with different spatial variabilities of the Manning's roughness fields were designed for a $2 \mathrm{D}$ flood modeling problem via the numerical solver (i.e. FLO-2D), within a rectangular modelling domain involving multiple channels. Twelve gPC/KLE models (i.e., M1-M12) with different combinations were built and the simulated moments were compared with those from Monte Carlo simulations. Further comparison between gPC/KLE and $\mathrm{PCM} / \mathrm{KLE}$ were conducted.

The study results revealed that a relatively higher accuracy in reproducing the statistics of the non-linear flood flow field could be achieved at an economical computational cost compared with traditional MC simulation and normal PCM/KLE approach. It was also indicated that: (i) the gPC/KLE model should be constructed using different number of SSG nodes (namely lower computational requirement) for spatial conditions with different levels of complexities; (ii) at the same $\sigma_{Z}^{2}$ level, the order of gPC approximation could also cause considerable effect on fitting performance without additional computational runs; and (iii) the spatial variability in the $\mathrm{x}$ and $\mathrm{y}$ directions would bring different effects on the predicted STDs of the flood flows, especially for those asymmetric geological conditions (i.e. multi-channel conditions).

The major contributions of this study are: (i) introduction of gPC/KLE to a two-dimensional flood inundation problem to address a heterogeneous random input field of logarithmic Manning's roughness involving different levels of spatial variability at reduced computational requirements; and (ii) evaluation of effects from adopting different numbers of eigenpairs in $x$ and $y$ coordinates considering existence of different levels of spatial variability associated with input random field. A number of limitations will need to be enhanced in the future. Firstly, flood modeling for many real-world cases may involve temporal uncertainty in model parameters, such as rainfall and inflow hydrographs; this was not tackled in this study. Furthermore, when other modeling/external processes, such as additional uncertainty sources, climate change impact, and hydrological process, are linked with flood modeling, the efficiency and configuration of the uncertainty assessment framework may need to be re-evaluated.

Acknowledgement: This research was supported by the National Key Research and Development Plan (2016YFA0601502, 2016YFC0502800), the Singapore's Ministry of Education (MOE) AcRF Tier 1
Project (Ref No. RG188/14; WBS no.: M4011420.030), and in part by the Land and Liveability National Innovation Challenge under L2 NIC Award No. L2NICCFP1-2013-3 (WBS no.: M4061545.D63). The authors deeply appreciate the reviewers' insightful comments and suggestions, which have significantly helped improve the manuscript.

\section{References}

Ahmadi, A., Moridi, A., and Han, D. (2015). Uncertainty assessment in environmental risk through Bayesian netowrks. J. Environ. Inf., 25(1), 46-59. http://dx.doi.org/10.3808/jei.20150 0294

Aronica, G., Bates, P.D., and Horritt, M.S. (2002). Assessing the uncertainty in distributed model predictions using observed binary pattern information within GLUE. Hydrol. Process., 16 (10), 20012016. http://dx.doi.org/10.1002/hyp.398

Assumaning, G.A., and Chang, S.Y. (2014). State and Parameter Estimation in Three-dimensional subsurface contaminant transport modeling using kalman filter coupled with monte carlo sampling. $J$. Environ. Inf., 24(2), 80-89. http://dx.doi.org/10.3808/ jei.2014002 80

Ballio, F., and Guadagnini, A. (2004). Convergence assessment of numerical Monte Carlo simulations in groundwater hydrology. Water Resour. Res., 40(4). http://dx.doi.org/10.1029/2003WR002876

Beven, K. (2006). A manifesto for the equifinality thesis. J. Hydrol., 320(1-2), 18-36. http://dx.doi.org/10.1016/j.jhydrol. 2005.07.007

Cai, Y.P., Huang, G.H., Yang, Z.F. and Tan, Q. (2009). Identification of optimal strategies for energy management systems planning under multiple uncertainties, Applied Energy, 86(4), 480-495. https://doi.org/10.1016/j.apenergy.2008.09.025

Cai, Y.P., Huang, G.H., Yang, Z.F., Lin, Q.G., and Tan, Q. (2009). Community-scale renewable energy systems planning under uncertainty - An interval chance-constrained programming approach. Renew. Sust. Energ. Rev., 13(4), 721-735. https://doi.org/ 10.1016/j.rser.2008.01.008

Chen, X., Huang, G., An, C., Yao, Y., \& Zhao, S. (2017). Emerging Nnitrosamines and $\mathrm{N}$-nitramines from Amine-based Post-combustion $\mathrm{CO}_{2}$ Capture - A Review. Chem. Eng. J., https://doi.org/10.1016/j. cej.2017.11.032

Chow, V.T., Maidment, D.R., and Mays, L.W. (1988). Applied hydrology, New York: McGraw-Hill.

Fan, Y., Huang, W., Huang, G., Huang, K., and Zhou, X. (2015). A PCM-based stochastic hydrological model for uncertainty quantification in watershed systems. Stochastic Environ. Res. Risk Assess., 29(3), 915-927. http://dx.doi.org/10.1007/s00477-014-095 4-8

Fan, Y.R. and Huang, G.H., A robust two-step method for solving interval linear programming problems within an environmental management context, J. Environ. Inf., 19(1), 1-9 (2012). http://dx. doi.org/10.3808/jei.201200203

FLO-2D Software (2012). FLO-2D Reference Manual 2009. https:/ /www.flo-2d.com/download/

Genz, A., and Keister, B.D. (1996). Fully symmetric interpolatory rules for multiple integrals over infinite regions with Gaussian weight. J. Comput. Appl. Math., 71(2), 299-309. http://dx.doi.org/ 10.1016/0377-0427(95)00232-4

Ghanem, R.G., and Spanos, P.D. (1991). Stochastic Finite Elements: A Spectral Approach, New York, NY: Springer New York. http:// dx.doi.org/10.1007/978-1-4612-3094-6

He, L., Wang, G.Q. and Zhang, C. (2012). Application of Loosely Coupled Watershed Model and Channel Model in Yellow River, China. Journal of Environmental Informatics, 19(1), 30-37. http:// dx.doi.org/10.3808/jei.201200206

Huang, G.H., and Cao, M.F. (2011). Analysis of Solution Methods for 
Interval Linear Programming. J. Environ. Inf., 17(2). http://dx.doi. org/ 10.3808/jei.201100187

Huang, S., Mahadevan, S., and Rebba, R. (2007). Collocation-based stochastic finite element analysis from random field problems. Probabilist. Eng. Mech., 22(2), 194-205. http://dx.doi.org/10.1016/ j.probengmech.2006.11.004

Huang, Y., and Qin, X.S. (2014a). Probabilistic collocation method for uncertainty analysis of soil infiltration in flood modelling. In 11th National Conference on Hydraulics in Civil Engineering \& 5th International Symposium on Hydraulic Structures: Hydraulic Structures and Society-Engineering Challenges and Extremes (p. 229), Engineers Australia. http://dx. doi.org/10.14264/uq1.2014.40

Huang, Y., and Qin, X.S. (2014b). Uncertainty analysis for flood inundation modelling with a random floodplain roughness field. Environ. Syst. Res., 3(1), 1-7. http://dx.doi.org/10.1186/2193-26973-9

Hunter, N.M., Bates, P.D., Horritt, M.S., and Wilson, M.D. (2007), Simple spatially-distributed models for predicting flood inundation: A review. Geomorphology, 90(3-4), 208-225. http://dx.doi.org/ 10.1016/j.geomorph.2006.10.021

Isukapalli, S.S., Roy, A. and Georgopoulos, P.G. (1998). Stochastic Response Surface Methods (SRSMs) for uncertainty propagation: Application to environmental and biological systems. Risk Anal., 18(3), 351-363. http://dx.doi.org/10.1111/j.1539-6924.1998.tb013 01.x

Jakeman, J., Eldred, M., and Xiu, D. (2010). Numerical approach for quantification of epistemic uncertainty. J. Comput. Phys., 229(12), 4648-4663. http://dx.doi.org/10.1016/j.jcp.2010.03.0 03

Karunanithi, N., Grenney, W.J., Whitley, D., and Bovee, K. (1994). Neural networks for river flow prediction. J. Comput. Civil. Eng., 8(2), 201-20. http://dx.doi.org/10.1061/(ASCE) 0887-3801(1994)8: 2(201)

Li, D., Chen, Y., Lu, W., and Zhou, C. (2011). Stochastic response surface method for reliability analysis of rock slopes involving correlated non-normal variables. Comput. Geotech., 38(1), 58-68. http://dx.doi.org/10.1016/j.compgeo.2010.10.00 6

Li, H., and Zhang, D. (2007). Probabilistic collocation method for flow in porous media: Comparisons with other stochastic methods. Water Resour. Res., 43(9). http://dx.doi.org/10.1029/2006wr005673

Li, L.B., Phoon, K.K., and Quek, S.T. (2007). Comparison between Karhunen-Loève expansion and translation-based simulation of non-Gaussian processes. Comput. Struct., 85(5-6), 264-276. http:// dx.doi.org/10.1016/j.compstruc.2006.10.010

Li, Y.P., Huang, G.H., Huang, Y.F., and Zhou, H.D. (2009). A multistage fuzzy-stochastic programming model for supporting sustainable water resources allocation and management. Environ. Model. Software, 24(7), 786-797. https://doi.org/10. 1016/j.envsoft. 2008.11.008

Li, Y.P., and Huang, G.H. (2009). Fuzzy-stochastic-based violation analysis method for planning water resources management systems with uncertain information. Information Sciences, 179(24), 42614276. https://doi.org/10.1016/j.ins.2009.09.001

Li, Z., Huang, G.H., Fan, Y.R. and Xu, J.L. (2015). Hydrologic Risk Analysis for Nonstationary Streamflow Records under Uncertainty. J. Environ. Inf., 26(1): 41-51. http://dx.doi. org/10.3808/jei.2015 00321

Lin, G., and. Tartakovsky, A.M. (2009). An efficient, high-order probabilistic collocation method on sparse grids for three- dimensional flow and solute transport in randomly heterogeneous porous media. $A d v$. Water Resour., 32(5), 712-722. http://dx.doi.org/10.1016/j. advwatres.2008.09.003

Liu, H., and Wang, D.Z.W. (2015). Global optimization method for network design problem with stochastic user equilibrium. Trans. Res. Part B, 72, 20-39. http://dx.doi.org/10.1016/j.trb. 2014.10.009

Marzouk, Y.M., Najm, H.N., and Rahn, L.A. (2007). Stochastic spectral methods for efficient Bayesian solution of inverse problems.
J. Comput. Phys., 224(2), 560-586. http://dx.doi.org/10.1016/j.jcp. 2006.10.010

O'Brien, J.S., Julien, P.Y., and Fullerton, W.T. (1993). Two- dimensional water flood and mudflow simulation. J. Hydraul. Eng., 119(2), 244-261.http://dx.doi.org/10.1061/(ASCE)0733-9429(1993) 119:2(244)

O'Connell, P., Nash, J., and Farrell, J. (1970). River flow forecasting through conceptual models part II-The Brosna catchment at Ferbane. J Hydro., 10: 317-29. http://dx.doi.org/10.1016/0022-1694(70)90 221-0

Pender, G., and Faulkner, H. (2011). Flood risk science and management Chichester, West Sussex, UK: Wiley-Blackwell, 2011.

Phoon, K.K., Huang, S.P., and Quek, S.T. (2002). Implementation of Karhunen-Loeve expansion for simulation using a wavelet-Galerkin scheme. Probabilist. Eng. Mech., 17(3), 293-303. http://dx.doi.org/ 10.1016/S0266-8920(02)00013-9

Qin, X.S., Huang, G.H., and Chakma, A. (2008). Modeling groundwater contamination under uncertainty: A factorial-design-based stochastic approach. J. Environ. Inf., 11(1), 11-20. http://dx.doi.org/ 10.3808/jei.200800106

Roy, R.V., and Grilli, S.T. (1997). Probabilistic analysis of flow in random porous media by stochastic boundary elements. Eng. Anal. Boundary Elements, 19(3), 239-255. http://dx.doi.org/10.1016/ S0955-7997(97)00009-X

Schwab, C., and Todor, R.A. (2006). Karhunen-Loève approximation of random fields by generalized fast multipole methods. J. Comput. Phys., 217(1), 100-122. http://dx.doi.org/10.1016/j.jcp.2006.01.048

Shen, J., Huang, G., An, C., Xin, X., Huang, C., \& Rosendahl, S. (2018). Removal of Tetrabromobisphenol A by adsorption on pinecone-derived activated charcoals: Synchrotron FTIR, kinetics and surface functionality analyses. Bioresource Technology, 247, 812820. https://doi.org/10.1016/j.biortech.2017.09.177

Shi, L.S., Yang, J.Z., Zhang, D.X., and Li, H. (2009). Probabilistic collocation method for unconfined flow in heterogeneous media. $J$. Hydrol., 365(1-2), 4-10. http://dx.doi.org/10.1016/j.jhydrol.2008.11. 012

Shi, L.S., Zhang, D.X., Lin, L., and Yang, J.Z. (2010). A multiscale probabilistic collocation method for subsurface flow in heterogeneous media. Water Resour. Res., 46(11), W11562. http://dx.doi. org/10.1029/2010wr009066.

Smolyak, S.A. (1963). Quadrature and interpolation formulas for tensor products of certain classes of functions. Dokl. Akad. Nauk SSSR, 4(240-243), 123.

Van Vuren, S., De Vriend, H., Ouwerkerk, S., and Kok, M. (2005). Stochastic modelling of the impact of flood protection measures along the river waal in the Netherlands. Nat. Hazards, 36(1), 81-102. http://dx.doi.org/10.1007/s11069-004-45 43-x

Wang, L., Huang, G., Wang, X., \& Zhu, H. (2018). Risk-based electric power system planning for climate change mitigation through multistage joint-probabilistic left-hand-side chance-constrained fracional programming: A Canadian case study. Renew. Sust. Energ. Rev., 82, 1056-1067. https://doi.org/10.1016/j.rser.2017. 09.098

Wang, X.H. and Du, C.M. (2003). An Internet Based Flood Warning System. J. Environ. Inf., 2(1): 48-56. http://dx.doi.org/10.3808/jei. 200300017

Wiener, S. (1938). The Homogeneous Chaos. Am. J. Math., 60(4), 897936. http://dx.doi.org/10.2307/2371268

Wu, C. B., Huang, G. H., Liu, Z. P., Zhen, J. L., and Yin, J. G. (2017). Scenario analysis of carbon emissions' anti-driving effect on Qingdao's energy structure adjustment with an optimization model, Part I: Carbon emissions peak value prediction. J. Environ. Manag., 172, 466-474. https://doi.org/10.1016/j. jclepro.2017. 10.216

Xiu, D. (2007). Efficient collocational approach for parametric uncertainty analysis. Commun. Comput. Phys., 2(2), 293-309.

Xiu, D. (2010). Numerical methods for stochastic computations: a spectral method approach, Princeton University Press. 
Xiu, D., and Karniadakis, G.E. (2002). The Wiener-Askey polynomial chaos for stochastic differential equations. SIAM J. Sci. Comput., 24(2), 619-644. http://dx.doi.org/10.1137/S10648275013 87826

Xiu, D., and Karniadakis, G.E. (2003). Modeling uncertainty in flow simulations via generalized polynomial chaos. J. Comput. Phys., 187(1), 137-167. http://dx.doi.org/10.1016/S0021-9991 (03)00092-5

Xiu, D., and Hesthaven, J. (2005). High-order collocation methods for differential equations with random inputs. SIAM J. Sci. Comput., 27(3), 1118-1139. http://dx.doi.org/10.1 137/040615201

Yang, W., and Yang, Z.F. (2014). Evaluation of Sustainable Environmental Flows Based on the Valuation of Ecosystem Services: A Case Study for the Baiyangdian Wetland, China. J. Environ. Inf., 24(2): 90-100. http://dx.doi.org/10.3808/jei.201400276

Yu, J.J., Qin, X.S., and Larsen, O. (2013). Joint Monte Carlo and possibilistic simulation for flood damage assessment. Stochastic Environ. Res. Risk Assess., 27(3), 725-735. http://dx.doi.org/10. 1007/s00477-012-0635-4

Yildirim, B., and Karniadakis, G.E. (2015). Stochastic simulations of ocean waves: An uncertainty quantification study. Ocean Model., 86, 15-35. http://dx.doi.org/10.1016/j.ocemod. 2014.12.001
Zhang, X.H., Ishidaira, H., Takeuchi, K., Xu, Z.X. and Zhang, X.W. (2004). An Approach to Inundation Simulation in Large River Basins Using the Triangle Finite Difference Method. J. Environ. Inf., 3(1). 51-63. http://dx.doi.org/10.3808/ jei.200400027

Zhao, X.U., Yang, H., Yang, Z., Chen, B., and Qin, Y. (2010). Applying the input-output method to account for water footprint and virtual water trade in the Haihe River basin in China. Environ. Sci. Technol., 44(23), 9150-9156. http://dx.doi.org/10. 1021/es100886r

Zhao, X., Chen, B., and Yang, Z. F. (2009). National water footprint in an input-output framework - a case study of China 2002. Ecol. Modell., 220(2), 245-253. https://doi.org/10.1016/j.ecolmodel.2008. 09.016

Zhang, D., and Lu, Z. (2004). An efficient, high-order perturbation approach for flow in random porous media via Karhunen-Loève and polynomial expansions. J. Comput. Phys., 194(2), 773-794. http:// dx.doi.org/10.1016/j.jcp.2003.09.015

Zheng, Y., Wang, W., Han, F., and Ping, J. (2011). Uncertainty assessment for watershed water quality modeling: a probabilistic collocation method based approach. Adv. Water Res., 34(7), 887-898. http://dx.doi.org/10.1016/j.advwatres.2011.0 4.016 